\title{
Final Report - Hybrid Monte Carlo-Deterministic Methods for Nuclear Reactor-Related Criticality Calculations
}

DOE Project: DE-FG07-00ID13920

Principal Investigator: Edward W. Larsen, University of Michigan

Report Period: 5/1/2000 - 10/31/2003

\subsection{Project Summary}

The overall goal of this project is to develop, implement, and test new hybrid Monte Carlo-deterministic (or simply hybrid) methods for the more efficient and more accurate calculation of nuclear engineering criticality problems. These new methods will make use of two (philosophically and practically) very different techniques - the Monte Carlo technique, and the deterministic technique - which have been developed completely independently during the past 50 years. The concept of this proposal is to merge these two approaches and develop fundamentally new computational techniques that enhance the strengths of the individual Monte Carlo and deterministic approaches, while minimizing their weaknesses.

The Monte Carlo method does not suffer from the space-angle-energy grid truncation errors that affect deterministic schemes and is particularly advantageous for complex 3-D geometries. However, Monte Carlo criticality solutions have statistical errors, are error-prone in weakly coupled systems, and can be difficult to bias efficiently. This proposal aims to confront these difficulties by developing new "hybrid" schemes that use deterministic techniques to obtain more accurate Monte Carlo solutions. Two kinds of hybrid schemes have been developed along these lines for fixed-source neutron transport problems: (i) schemes that determine biasing parameters for the Monte Carlo simulation from approximate deterministic (usually, adjoint) calculations, and (ii) schemes that use more accurate functionals for desired responses, which make use of both deterministic (adjoint) and Monte Carlo (forward) information. Extending, testing, and optimizing these hybrid algorithms for criticality problems is the theoretical goal of this project. The practical goal is to achieve methods that can solve difficult, realistic Monte Carlo criticality problems more efficiently and reliably, and with significantly less user-input.

\subsection{Phase 1. Summary of Objectives and Accomplishments}

Phase 1 had a duration of 12 months. This phase covered the development of monoenergetic 1-D test codes to implement and test each of the basic concepts outlined below. The Phase 1 Performance Period was May 1, 2000 - April 30, 2001. Deliverable: A topical report at the end of Phase 1 to indicate: (i) the performance of the various methods outlined in the proposal for 1-D mono-energetic problems; (ii) whether a proof-of-principle has been developed.

\section{Objectives:}

The proposal will consider three main strategies for new hybrid methods: (i) a Variational approach, (ii) a Local Importance Function (LIFT) approach, and (iii) a Weight Windows 
approach. A fourth general approach - of advantageously combining concepts from three different approaches - was also proposed.

To describe the basic ideas underlying the variational approach, we express the general criticality problem as

$$
L \psi=\frac{1}{k} F \psi,
$$

where $L$ is the streaming+collision-scattering operator, $F$ is the fission operator, $k$ is the criticality eigenvalue of the system, and $\psi$ is the eigenfunction. The simplified notation in Eq. (1) conceals a wealth of detail and complexity. Accurately and efficiently solving solving this equation for the eigenvalue $k$, for realistic problems, is the goal of this project. This eigenvalue problem, and other problems like it, lies at the root of nuclear engineering design and analysis.

If the inner product of two functions $\psi^{*}$ and $\psi$ is represented by

$$
\left(\psi^{*}, \psi\right)=\int_{0}^{\infty} \int_{4 \pi} \int_{V} \psi^{*}(\underline{r}, \underline{\Omega}, E) \psi(\underline{r}, \underline{\Omega}, E) d V d \Omega d E
$$

then adjoint operators $L^{*}$ and $F^{*}$ can be defined satisfying $\left(\psi^{*}, L \psi\right)=\left(L^{*} \psi^{*}, \psi\right)$ and $\left(\psi^{*}, F \psi\right)=\left(F^{*} \psi^{*}, \psi\right)$, and the adjoint problem to Eq. (1) is:

$$
L^{*} \psi^{*}=\frac{1}{k} F^{*} \psi^{*}
$$

Multiplying Eq. (1) by an arbitrary function $g(\underline{r})$ and integrating over space, angle, and energy, we get:

$$
\frac{1}{k}=\frac{(g, L \psi)}{(g, F \psi)} \text {. }
$$

This equation is a main ingredient of conventional Monte Carlo simulations. Specifically, the function $g$ is taken to be equal to unity, while the function $\psi$ is obtained by simulating a very large number of particle histories and introducing the results of each history into the functional on the right side of Eq. (4). It is easy to show that if the Monte Carlo estimate $\Psi$ of the exact eigenfunction $\psi$ has error of $O(\varepsilon)$, then the error resulting from using $\Psi$ in Eq. (4) is also $O(\varepsilon)$ :

$$
\frac{(g, L \Psi)}{(g, F \Psi)}=\frac{1}{k}+O(\varepsilon)
$$

The variational approach to hybrid Monte Carlo simulations makes use of a more sophisticated result. Let $\Psi$ be an estimate of $\psi$ [the solution of Eq. (1)] with an error of $O(\varepsilon)$, and let $\Psi^{*}$ be an estimate of $\psi^{*}$ [the solution of Eq. (3)], with an error of $O(\delta)$. Then, it can be shown that

$$
\frac{\left(\Psi^{*}, L \Psi\right)}{\left(\Psi^{*}, F \Psi\right)}=\frac{1}{k}+O(\varepsilon) O(\delta) .
$$

Thus, by having "first-order" estimates of both the adjoint and forward eigenfunction, one can achieve a much more accurate "second-order" estimate of $k$. In the variational variance reduction 
(VVR) method, an inexpensive deterministic (typically a diffusion) calculation is performed to obtain a deterministic estimate $\Psi^{*}$, defined on a grid; then one evaluates $\Psi$ using Monte Carlo, and both estimates are introduced into the variational functional [left side of Eq. (6)]. The resulting calculation is more complicated, for several reasons: (1) Conventionally, the simple but relatively poor estimate $\Psi^{*}=g=1$ is used. In the variational approach, $\Psi^{*}$ is expected to be a reasonably accurate representation of the adjoint eigenfunction $\psi^{*}$. Extra cost is associated with calculating this $\Psi^{*}$; (2) After calculating $\Psi^{*}$ and introducing it into the variational functional [left side of Eq. (6)], a reduced functional results, which depends on the specific pointwise representation of $\Psi^{*}$. This reduced functional is more costly to evaluate using a Monte Carlo estimate of $\psi$ than the standard functional, based on $\Psi^{*}=g=1$. Thus, extra time and cost (per particle history) must be spent evaluating the variational functional.

However, the variational functional [left side of Eq. (6)] is expected to produce a more accurate estimate of $k$ using fewer Monte Carlo particles than the standard functional [left side of Eq. (5)]. If the variational functional is sufficiently accurate, then given the same computing resources (time), it will produce a more accurate estimate of $k$ than the standard functional. However, only evaluation and testing can determine if the increased accuracy of the variational functional outweighs the extra cost (per particle) of evaluating it.

Two other approaches were to be considered: the Local Importance Function Transform (LIFT), and the Weight Window (WW) approaches. In both of these approaches, the forward Monte Carlo process is modified in a nonanalog way. In the LIFT approach, particles have a weight, and the "rules of the Monte Carlo game" are modified - the particle mean free path and the direction of flight after a scattering event are altered (in a way that introduces no error or bias into the estimate of $k$ ) to more advantageously "steer" Monte Carlo particles in phase space.

In the WW approach, a different nonanalog scheme is employed. Now, at each location in space, an upper and a lower weight are assigned, and all Monte Carlo particles at that location will have a weight that lies between these specified upper and lower bounds. If a particle streams into a location with a weight that is too high, it is split; if the weight is too low, the particle is Russian-Rouletted.

The VVR approach outlined above does not require Monte Carlo particles to be processed in a nonanalog fashion - but it permits this. The LIFT and WW approaches do require a nonanalog treatment of Monte Carlo particles. The LIFT and WW approaches use the same estimate of the adjoint $\Psi^{*}$ as in the VVR method; the LIFT method employs $\Psi^{*}$ to determine exactly how to alter the mean free path and scattering angle estimates; while the WW technique employs $\Psi^{*}$ to define the upper and lower weight windows.

Performing the testing and evaluation of these concepts in 1-D was the goal of phase 1 of this project.

\section{Accomplishments:}

The fine details of the VVR method, the LIFT method, and the WW method were decided upon, and a 1-D monoenergetic code was written to implement, test, and compare these various concepts.

Our testing showed that in 1-D, the VVR concept was extremely beneficial, showing huge gains (often well over a factor of 100) over conventional Monte Carlo estimates. The VVR 
method is particularly well-suited for diffusive eigenvalue problems, characteristic of nuclear reactors. One reason is that an inexpensive adjoint calculation based on diffusion theory (rather than transport theory) is appropriate; this is the strategy that we adopted. This helped minimize the extra cost of obtaining a deterministic estimate of $\Psi^{*}$. Also, for thick, diffusive problems, the angular dependence of the adjoint (and forward) angular fluxes is weak, which enabled us to use a simple first-order spherical harmonic representation of $\psi^{*}$.

Unfortunately, we did not observe such gains with the LIFT and WW approach. Investigations into the reasons for this suggested the following. (1) Our focus was on eigenvalue problems for relatively diffusive (highly scattering, optically thick) systems. In such problems, the LIFT method alters the rules of the Monte Carlo game by only a small amount, but the cost of implementation is not insignificant. Overall, we observed that the cost of implementing LIFT outweighed its marginal benefits for this class of diffusive problems. (2) The WW method also increased the cost of the calculation (per particle history), with insufficient gain in accuracy to yield an overall computational benefit.

We also tried using LIFT and WW with VVR. Again we found that in general, the extra calculational expense of LIFT and WW was not overcome by a sufficient increase in the accuracy of the estimate of $k$ to warrant the use of these techniques with VVR.

Our results were written up and presented at several ANS meetings. Reference [1] described some preliminary work in which the VVR concept was applied - with success - to source-detector problems. Ref. [2] described early 1-D work on implementing and testing the VVR concept for criticality problems. Ref. [3] is theoretical in nature - it describes how the VVR concept relates to a new class of zero-variance methods. (The VVR method can be understood theoretically as a practical low-variance approximation to an idealized zero-variance method.) Ref. [4] describes the 1-D multigroup VVR method with extensive numerical results. In relatively simple, homogeneous-medium problems, the VVR method using a $\mathrm{P}_{1}$ adjoint yielded gains in the FOM ranging from the factors 10-1,000; using a $\mathrm{P}_{3}$ adjoint, gains in the FOM ranged from the factors 20-3,500. For heterogeneous-media problems, the VVR method using a $\mathrm{P}_{1}$ adjoint yielded gains in the FOM ranging from the factors 10-50; using a $\mathrm{P}_{3}$ adjoint, gains in the FOM ranged from the factors 60-170. In the very worst cases, the speedups in the calculations using VVR were a factor of 10, and in most cases were much higher. These results were very encouraging. They showed that the principle of using a Monte Carlo scheme that is more costly per particle, but that employs a more accurate functional to estimate the quantity of interest, can lead to greatly reduced run times because of the fewer number of Monte Carlo particles needed to achieve the desired accuracy.

\subsection{Phase 2. Summary of Objectives and Accomplishments}

Phase 2 had a duration of 12 months. This phase covered the extension and testing of the methods developed in Phase 1 into multidimensional geometries. The Phase 2 performance period is May 1, 2001 - April 30, 2002. Deliverable: A topical report at the end of Phase 2 indicated the performance of the various methods in multidimensional geometries.

\section{Objectives:}


The objectives of this phase of the project were to develop a multidimensional code in which, guided by our 1-D results, we implement and test the most promising of the hybrid techniques considered in Phase 1.

\section{Accomplishments:}

We accomplished this goal and learned some interesting, but on hindsight not entirely unexpected, things. Our multidimensional 3-D code was based on a straightforward Cartesian grid in the three spatial variables $x, y$, and $z$. As in 1-D, we opted to use a diffusion code to estimate $\Psi^{*}$. Unlike the situation in 1-D, in which diffusion codes (at least in one group) can be solved directly by Gaussian Elimination, 3-D diffusion problems require iterative solutions. Thus, in 3-D geometries, the cost of obtaining the deterministic estimate of $\Psi^{*}$ is relatively greater than in 1-D. Nevertheless, this cost was still minor, compared to the cost of the Monte Carlo part of the simulation.

We also developed a generalization to the hybrid VVR technique, in which enhanced estimates of the eigenfunction $\psi$ can be obtained. This requires an entirely different variational approach, but which conceptually fits into the already-developed VVR technique for estimating the eigenvalue $k$.

In addition, we began the implementation and testing of the concept of using an estimate of the adjoint eigenfunction $\psi^{*}$ which is not obtained from a deterministic calculation, but rather from a Monte Carlo calculation. In general, this procedure does not yield a method that is more efficient than the original VVR method in which $\psi^{*}$ is estimated deterministically. Nevertheless, estimating $\psi^{*}$ using Monte Carlo has certain practical advantages:

- The Monte Carlo adjoint VVR method is generally more efficient than the conventional Monte Carlo method for estimating $k$. Thus - without comparisons to the deterministic adjoint VVR method - the Monte Carlo adjoint VVR method represents generally a gain in efficiency.

- Developing a Monte Carlo-only VVR code will be easier and require less programming effort than developing a pair of codes (one deterministic, one Monte Carlo) that must be run in tandem, or one dual-purpose code containing both deterministic and Monte Carlo methods.

- A Monte Carlo-only VVR code would probably be more user-friendly. For instance, it would not require the user to understand the accuracy-limitations of a deterministic method that should be weighed in selecting a spatial grid. The user of such a code would not need to be familiar with both deterministic and Monte Carlo methods.

- For especially difficult problems, such as ones containing voids and streaming regions for which deterministic methods are prone to error - it may be advantageous to use a Monte Carlo adjoint in which one has confidence that the estimations will improve with computing time.

We wrote up our results and again presented them at an ANS meeting. References [5] and

[6] describe some of our early 3-D work. Ref. [5] presents the VVR method for obtaining 
improved estimates of eigenfunctions, while Ref. [6] describes our first experience in using the VVR method with adjoint eigenfunctions that are estimated by Monte Carlo (rather than deterministically).

\subsection{Phase 3. Summary of Objectives and Accomplishments}

Phase 3 also had a duration of 12 months. This phase covered the extension and testing of the multidimensional methods into full energy-dependent simulations. The Phase 3 deliverable was a final report indicating: (i) the performance of the various methods in multidimensional geometries with the energy-dependent cross sections, (ii) the overall worth of the technical hybrid Monte Carlo-deterministic concept as applied to 3-D, energy-dependent criticality problems.

\section{Objectives:}

Our goals for this phase of the project were to complete the three most promising elements of the VVR work that we had seen in our earlier research: extensions to full 3-D multigroup problems of (i) the original VVR method (using a deterministic estimate of the adjoint function) for estimating $k$, (ii) the adjoint VVR method (using a Monte Carlo estimate of the adjoint function) for estimating $k$, and (iii) the new VVR method for estimating the eigenfunction $\psi$.

\section{Accomplishments:}

We accomplished these goals and wrote one major conference paper [7] and two fulllength journal articles [8] and [9].

Reference [7] is an expanded version of Ref. [6], describing in greater detail - and with extra numerical results - the option of using the VVR method with a Monte Carlo rather than a deterministic estimate of $\Psi^{*}$. Ref [8] is a full-length journal version of [7]. Ref. [9] is a fulllength journal article describing the VVR method as originally conceived - as a hybrid method

in which $\Psi^{*}$ is estimated using a deterministic technique and $\Psi$ is estimated using Monte Carlo - for 3-D multigroup problems, with many numerical results. These results are very encouraging - gains in the FOM by factors of 10 to 40 are observed.

Our final comments and impressions of the results coming from this project are as follows.

1. The concept of merging deterministic and Monte Carlo techniques for $k$-eigenvalue problems, using the VVR techniques described above, is sound and beneficial. Due to lack of time and resources, our multigroup VVR technique could not be extended to the full continuous-energy dependence of production Monte Carlo codes such as MCNP. Nevertheless, it is very clear that significant gains in the efficiency of Monte Carlo criticality codes can be obtained by using the concepts developed in this project.

2. While the use of a hybrid Monte Carlo-deterministic method for $k$-eigenvalue problems is beneficial, it will require changes in procedure on the part of code users. The reason is 
that each given problem will have to be set up and run for both a deterministic and a Monte Carlo calculation. This will require the code user to be conversant with both Monte Carlo and deterministic methods, since (for example) choices will have to be made in defining a spatial grid for the deterministic calculation. However, this extra degree of expertise will be compensated by the fact that the Monte Carlo simulation will run much more efficiently.

3. A significant number of successful "hybrid" Monte Carlo-deterministic methods now exist for performing practical source-detector problems; a review article on this subject has recently been published [10]. In most of these techniques, a deterministic adjoint calculation is first performed, the results of which are used to (automatically) define biasing parameters for a subsequent nonanalog Monte Carlo calculation. The deterministic adjoint calculation in these techniques is aimed primarily at removing the task, from the Monte Carlo code user, of developing the multitude of biasing parameters that are needed to make Monte Carlo codes run for difficult source-detector problems. For difficult source-detector problems, determining these parameters can require hours, or even days - even for an experienced user. An added benefit is that computer-generated biasing parameters are often much more efficient than the ones obtained by humans. Thus, in effect, two birds are killed with one stone: (i) the task of obtaining Monte Carlo biasing parameters is taken over by the computer, and (ii) the computer-generated biasing parameters enable the Monte Carlo code to run more efficiently than the humangenerated ones. Thus, hybrid Monte Carlo-Deterministic methods are much more of a "black box" calculational tool than conventional Monte Carlo methods. These hybrid source-detector techniques are similar in many ways to the VVR method developed in this project: a deterministic adjoint calculation is first performed, and a subsequent Monte Carlo calculation is performed in a way which is made more efficient by the preliminary deterministic calculation. The work accomplished in this project is an extension of this idea to criticality problems - and to the use of a more accurate functional to calculate the quantity of interest - in our case, the eigenvalue $k$. If future computer codes are developed to facilitate the use of hybrid Monte Carlo-deterministic techniques for sourcedetector problems, then it is certain that similar codes could be developed to implement the VVR techniques developed in this project for $k$-eigenvalue problems.

\section{References \& Presentations:}

1. J.D. Densmore and E.W. Larsen, "A New Variational Variance Reduction Method for Monte Carlo Source-Detector Problems," Trans. Am. Nucl. Soc. 83, 336 (2000).

2. J.D. Densmore and E.W. Larsen, "Variational Variance Reduction for Monte Carlo Criticality Calculations," Trans. Am. Nucl. Soc. 84, 177 (2001).

3. E.W. Larsen and J.D. Densmore, "New Zero-Variance Methods for Monte Carlo Criticality and Source-Detector Problems," Trans. Am. Nucl. Soc. 84, 175 (2001). 
4. J.D. Densmore and E.W. Larsen, "Variational Variance Reduction for Monte Carlo Criticality Problems," Proc. ANS Topical Meeting: International Conference on Mathematical Methods to Nuclear Applications, September 9-13, 2001, Salt Lake City, Utah, CD (ISBN: 0-89448-661-6, ANS Order No. 700286) available from the American Nuclear Society, 555 N. Kensington Avenue, La Grange Park, IL 60525 (2001).

5. J.D. Densmore and E.W. Larsen, "Variational Variance Reduction for Monte Carlo Eigenfunction Problems," Trans. Am. Nucl. Soc. 87, 227 (2002).

6. J.D. Densmore and E.W. Larsen, "Use of Monte Carlo Adjoint Simulations in VVR Criticality Calculations," Trans. Am. Nucl. Soc. 87, 228 (2002).

7. J.D. Densmore and E.W. Larsen, "Variational Variance Reduction for Criticality Calculations Using Monte Carlo Adjoint Fluxes," Proc. ANS Topical Meeting: Nuclear, Mathematical, and Computational Sciences: A Century in Review - A Century Anew, April 6-10, 2003, Gatlinburg, Tennessee, CD-ROM (ISBN: 0-89448-674-6, ANS Order No. 700300) available from the American Nuclear Society, 555 N. Kensington Avenue, La Grange Park, IL 60525 (2003).

8. J.D. Densmore and E.W. Larsen, "Variational Variance Reduction for Particle Transport Eigenvalue Calculations Using Monte Carlo Adjoint Simulation,” J. Comp. Phys. 192, 387 (2003).

9. J.D. Densmore and E.W. Larsen, "Variational Variance Reduction for Monte Carlo Eigenvalue and Eigenfunction Problems," Nucl. Sci. Eng., to appear (2004).

10. A. Haghighat and J. Wagner, "Monte Carlo Variance Reduction with Deterministic Importance Functions," Prog. Nucl. Energy 42, 25 (2003). 